\title{
Control de inventarios y su aplicación en una compañía de telecomunicaciones
}

\author{
The application of inventory control in a \\ telecommunications company
}

\author{
C.A. Álvarez-Herrera, M. Cabrera-Ríos \\ División de Posgrado en Ingeniería de Sistemas, FIME-UANL \\ E-mails: carlos@yalma.fime.uanl.mx y mcabrera@mail.uanl.mx
}

(Recibido: enero de 2006; aceptado: marzo de 2007)

\begin{abstract}
Resumen
El uso de técnicas de un área partic ular del conocimiento para resolver problemas en otras áreas distintas es una contribución valiosa del trabajo interdisciplinario. En este trabajo se describe la solución a un problema de planeación de capacidad de una red de telecomunicaciones en una compañía, por medio de la utilización de técnicas de control de inventarios. Adicionalmente, se ilustra el comportamiento de las soluciones prescritas ante diversos escenarios. El método de solución resulta eficaz, sencillo y de fácil instauración.
\end{abstract}

Descriptores: Inventarios, telecomunicaciones, optimización, planeación de capacidad.

\begin{abstract}
The use of tech niques par tic u lar to a field to solve problems en coun tered in other different areas is a valuablecon tribu tion of in ter disciplinarywork. In this paper, the solution to a prob lem of ca pac ity plan ning in a tele com mu nica tions com pany is achieved through the use of Inven tory Con trol tech niques. Ad ditionally, the be hav ior of the prescribed solu tions is il lus trated with the aid of differ ent scenarios. The solu tion method is shown to be effec tive, sim ple and easy to im ple ment.
\end{abstract}

Keywords:Inven torycontrol, telecommunications, op timization, capacityplanning.

\section{Introducción}

Una red de telecomunicaciones está formada por una serie de estaciones de trabajo coordinadas por máquinas especiales, denominadas servidores, y por un conjunto variable de dispositivos autónomos, tales como routers y switches. Cada dispositivo activo que interviene en la comunicación de forma autónoma se denomina nodo. Todos ellos se comunican entre sí.

Los sistemas de comunicación en red se basan en la arquitectura cliente-servidor, que es una forma específica de diseño de apli- caciones (Sprangins et al., 1992). Así, el cliente es el ordenador que se encarga de efectuar una petición o solicitar un servicio, mientras que el servidor es el ordenador remoto que controla dichos servicios. Cada servicio solicitado por el cliente es medido por unidades de ancho de banda. Para que una red de telecomunicaciones pueda ejecutar los servicios de todos los clientes, debe contar con la capacidad suficiente disponible en términos de unidades de ancho de banda en sus nodos.

La importancia de la labor de planeación que debe realizarse para adquirir el equipo que provea las unidades de ancho de banda 
necesarias, tomando como base un pronóstico de demanda y una capacidad inicial instalada es evidente. El balance importante está en mantener un inventario mínimo de equipo que garantice un cierto nivel de servicio al cliente. En la Figura 1 se muestran los datos iniciales del problema: un pronóstico de demanda para un año particular y la capacidad inicial (Álvarez y Cabrera, 2005).

\section{Optimización en un problema de planeación de capacidad para una red de telecomunicaciones}

Al tratar el problema de planeación de capacidad en telecomunicaciones como un problema de optimización, se busca encontrar las cantidades de equipo a pedir en el tiempo, con el objetivo de minimizar el costo asociado con el inventario, y bajo la consideración de tratar de cumplir con ciertos niveles de demanda. Dado que los datos de la demanda provienen generalmente de un pronóstico, una práctica común es además definir un stock de seguridad para proteger contra las posibles fluctuaciones de la demanda real. Para resolver este problema, por lo general se recurre a un modelo de programación matemática. El modelo resultante, sin embargo, puede fácilmente volverse muy difícil de resolver al analizar una cantidad elevada de períodos (Coyle, 1998), pues la mayoría de los casos involucra variables binarias y enteras, definiendo así un problema combinatorio. Un modelo típico de planeación se puede encontrar en Álvarez y Cabrera (2005), y se reproduce a continuación para planear doce períodos con el objetivo de minimizar costo:

$$
\begin{aligned}
& \text { Encontrar } x_{i}, y_{i} \quad i=1,2, \ldots, 12 \text { para } \\
& \text { Minimizar } \sum_{i=1} a_{i} x_{i}+\sum_{i=1} b_{i} I_{i}+\sum_{i=1} f_{i} y_{i}
\end{aligned}
$$

Sujeto a:

$$
\begin{array}{ll}
c_{1}=c_{0} & \\
c_{i}+x_{i}-c_{i+1}=0 & i=1,2, \ldots 12 \\
c_{i} \geq D_{i} & i=2,3, \ldots 12 \\
C_{i}-I_{i}=D_{i} & i=1,2, \ldots 12 \\
x_{i}-M y_{i} \leq 0 & i=1,2, \ldots 12 \\
y_{i} \in\{0,1\} & i=1,2, \ldots 12 \\
x_{i} \in Z^{+} & I=1,2, \ldots 12
\end{array}
$$

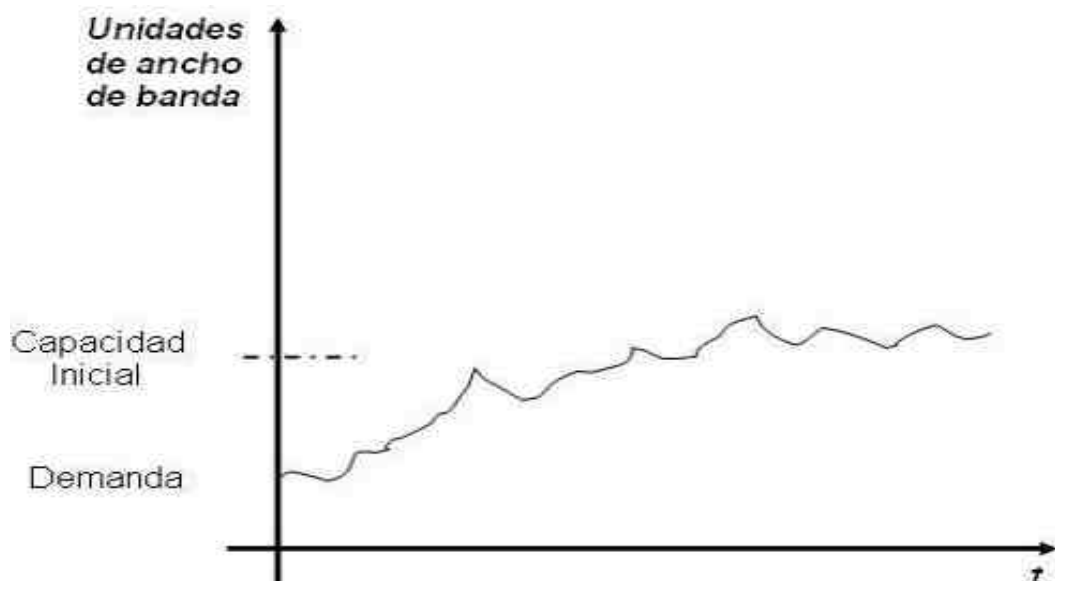

Figura 1. Pronóstico de demanda en una red de telecomunicaciones para un año específico 
Donde, para el $i$-ésimo período, $x_{i}$ representa la cantidad de unidades de ancho de banda requeridas, $I_{i}$ la cantidad de unidades de ancho de banda en inventario, $y_{i}$ es una variable binaria que toma el valor de 1 si se genera una orden de compra de equipo y 0 si no, y $C_{i}$ es la capacidad en unidades de ancho de banda; $a_{i}, b_{i}, \mathrm{y} f_{i}$ representan costos por unidad de ancho de banda ordenada, mantenida en inventario y el costo de generar una orden de compra respectivamente; $C_{0}$ es la capacidad inicial de ancho de banda y $M$ es un nú mero positivo muy grande.

La idea en este trabajo es resolver el problema de planeación de capacidad para una red de telecomunicaciones, planteado aquí como un problema de programación matemática entera, bajo un enfoque distinto: la utilización de técnicas de control de inventarios. Con el caso de estudio que se presenta a continuación y los múltiples escenarios, se intenta demostrar que utilizar estas técnicas puede generar soluciones competitvas en poco tiempo y a un costo computacional bajo.

\section{Enfoque de inventarios para un problema de telecomunicaciones}

Los inventarios, definidos de manera general, son aquellos artículos a la mano destinados para consumo. El control de inventarios está integrado por té cnicas para determinar cuándo deben reabastecerse los inventarios actuales y cuá nto debe reabastecerse (Solow y Mathur, 1996). El control de inventarios busca satisfacer las demandas de los clientes a un nivel predefinido con un bajo costo. Es claro, sin embargo, que estos objetivos se encuentran en conflicto. Una curva de inventarios en el tiempo se muestra gráficamente en la figura 2.

Por otro lado, el propósito inicial en la industria de telecomunicaciones es brindar servicio a todos sus clientes, aumentando la capacidad cuando se requiera. La capacidad en este contexto es la cantidad de unidades de ancho de banda disponible para dar servicio a los clientes (Figura 3).

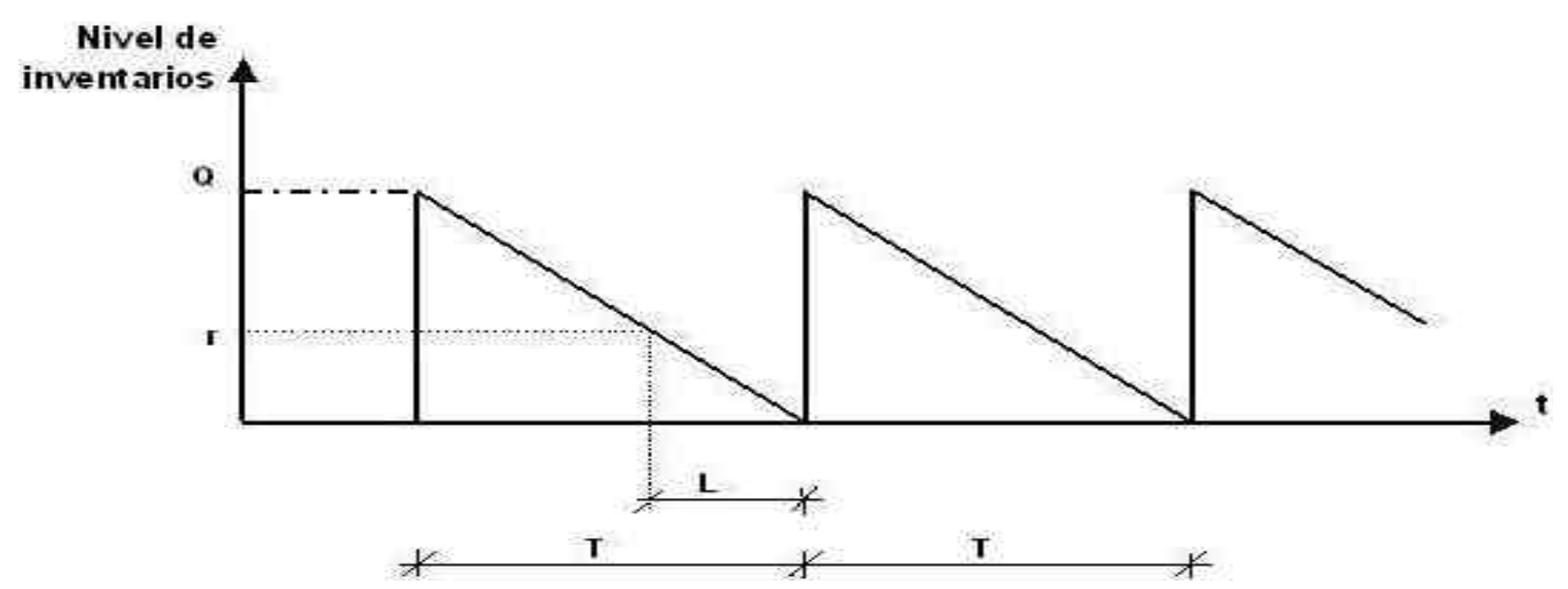

Figura 2. Evolución de inventario: Al inicio de un periodo de tiempo (T) se tiene un nivel de inventarios con una cantidad $(Q)$ de artículos, conforme transcurre el tiempo el nivel de inventarios disminuye hasta llegar a un punto ( $r)$, el cual nos indica el momento en el que tenemos que reordenar el inventario, tomando en cuenta un tiempo guia $(L)$ que va desde que se ordenan los artículos hasta que se tienen disponibles 


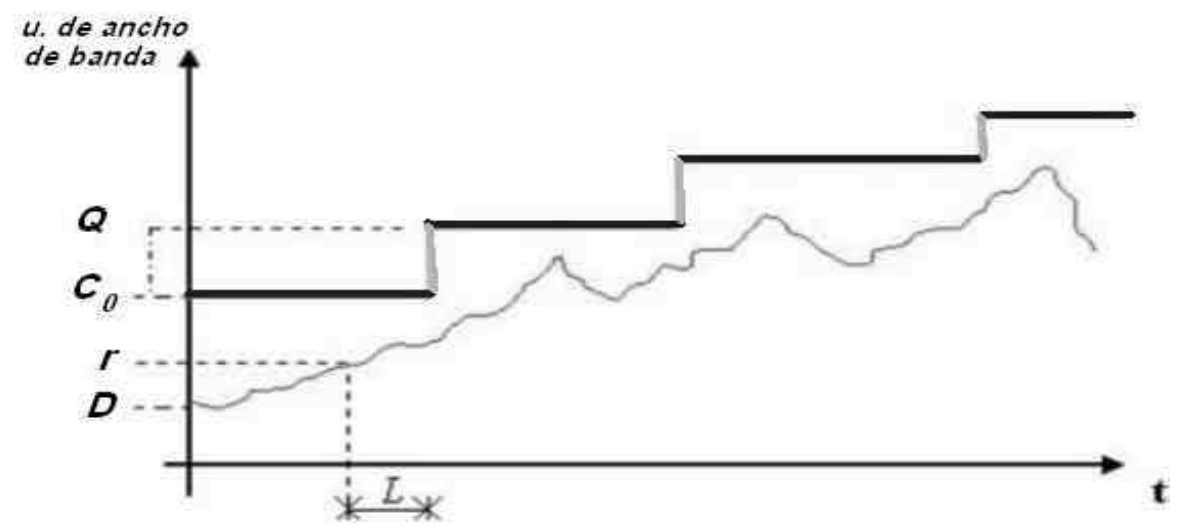

Figura 3. Evolución de la Demanda (D) y la Capacidad (C) en el tiempo para una red de telecomunicaciones: Se tiene una Capacidad inicial $\left(C_{0}\right)$ con la cual se abastecerá la demanda, conforme transcurre el tiempo la demanda crece y se define un punto de reorden ( $r$ ) en el cual se pedirá una cantidad $(Q)$ de unidades de ancho de banda, tomando en cuenta un tiempo guía $(L)$

En control de inventarios se busca entonces adaptar la oferta a los diferentes niveles de la demanda determinada por el consumo de los clientes. El inventario en un período determinado se da por la diferencia matemática entre la cantidad de productos que se tienen disponibles para el consumo (oferta) y la cantidad de productos que los clientes consumen (demanda). En telecomunicaciones, la diferencia matemática entre la capacidad instalada (oferta) y la utilización (de- manda), determina la capacidad ociosa, y se traduce así en el nivel de inventarios de la red. Al graficar la evolución de la diferencia entre Capacidad y Demanda en el tiempo, obtenemos una gráfica muy similar a la de control de inventarios como se muestra en la figura 4. Con tales similitudes, es entonces posible utilizar la teoría de control de inventarios para dar solución al problema de planeación de capacidad en redes de telecomunicaciones.

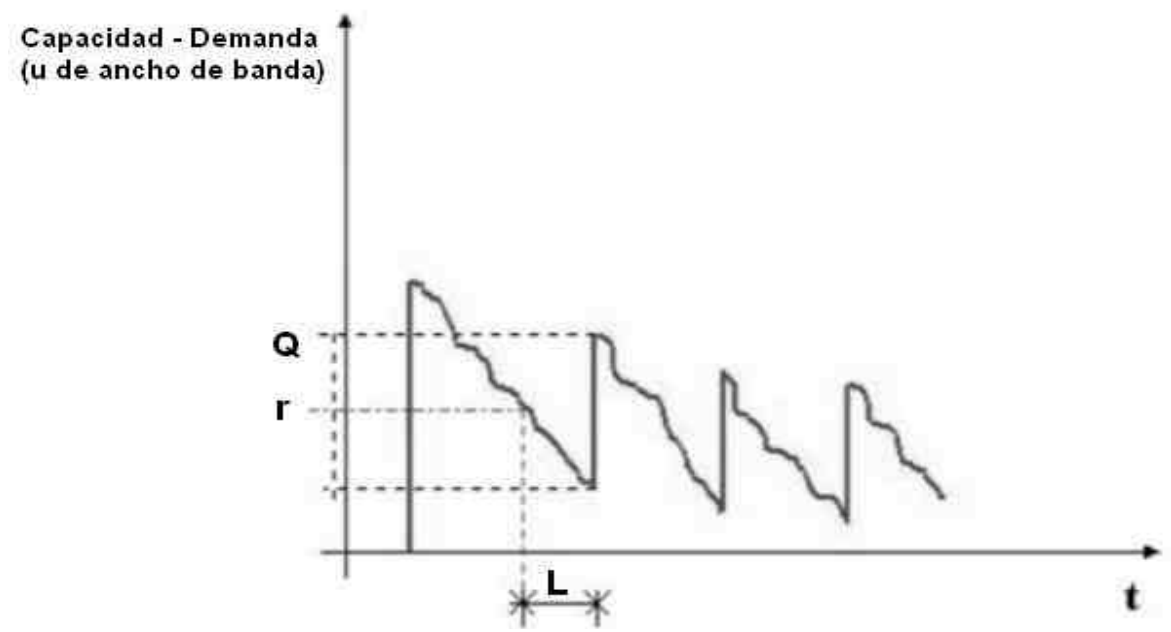

Figura 4. Evolución de (Capacidad-Demanda) en el Tiempo: Se tiene un nivel de inventarios con una cantidad $(Q)$ de unidades de ancho de banda, conforme transcurre el tiempo el nivel de inventarios disminuye hasta llegar a un punto ( $r)$, el cual nos indica el momento en el que tenemos que reordenar, tomando en cuenta un tiempo guía (L) 
La aplicación de varios modelos de inventario en el problema de planeación fue explorada en Álvarez y Cabrera-Ríos (2005). La evaluación de estos modelos arrojó que es plausible y recomendable utilizar el Modelo de Nivel de Servicio, en donde el usuario establece un cierto nivel de servicio que quiere proveer a sus clientes dada una demanda estocástica. El nivel de inventario en este modelo se monitorea continuamente. El modelo determina el nivel de inventario en el cual se debe generar una orden (punto de reorden) de manera tal, que exista un cierto nivel de garantía para no quedarse sin inventario durante el tiempo guía. Esta garantía está asociada con el nivel de servicio predefinido para el cliente. Una de las condiciones importantes para este modelo es que la demanda siga una distribución normal.

En la siguiente sección se presenta una aplicación real de las ideas propuestas en este apartado. Posteriormente, se presentan dos escenarios teóricos que permitieron probar el método.

\section{Caso de estudio}

En este estudio se analizaron datos históricos reales de capacidad y demanda de 5 añ os en una red de telecomunicaciones. La capacidad inicial de la red es de 128 unidades y la demanda máxima durante los 5 añ os es de 709 unidades de ancho de banda para un período específico. Se utilizó un modelo de nivel de servicio para determinar cómo se debía haber comprado equipo durante este horizonte de 5 años. Durante el análisis se utilizó el tiempo guía conocido y prácticamente constante de 4 meses de un proveedor real.

Se utilizó en este caso un nivel de seguridad de $80 \%$. Al aplicar el modelo, despué s del primer pedido se cumplió con la demanda en todos los períodos (Figura 5). El inventario se mantuvo alto debido a que el objetivo de esta té cnica es garantizar el cumplimiento de la demanda; sin embargo, el método permite al usuario variar el nivel de servicio para permitir actitudes menos conservadoras en el nivel de inventario, si así se desea.

El modelo habría prescrito ordenar un total de 952 unidades distribuidas en los 5 años, terminando con una capacidad final de 1080 unidades. No se habrían podido satisfacer 16 unidades de demanda en estos 5 años: 4 unidades en el 2o. mes y 12 unidades en el 3er. mes. Esto se debe a que en tales períodos el tiempo guía para surtir el primer pedido es de 4 meses, quedando así sin protección inicial.

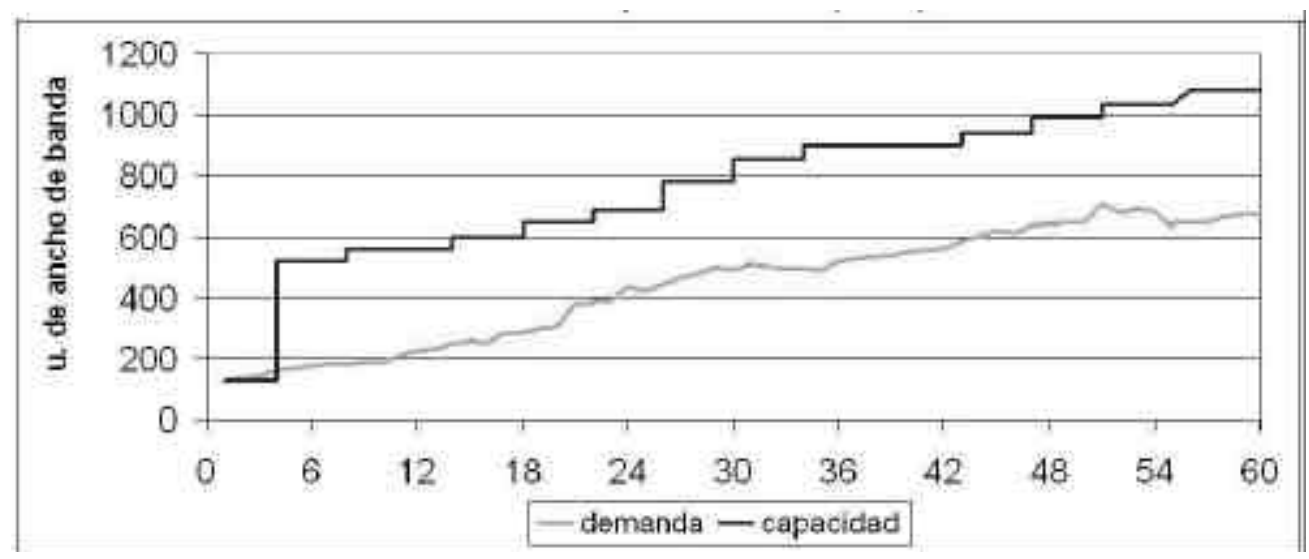

Figura 5: Evolución de la demanda y la capacidad durante 5 años utilizando un modelo de Nivel de Servicio de $80 \%$ 
Control de inventarios y su aplicación en una compañía de telecomunicaciones

El supuesto de normalidad de la demanda no se cumple en este caso; sin embargo, observamos que la solución actuó satisfactoriamente ante las fluctuaciones de demanda. Este aspecto requerirá de mayor estudio en el futuro para determinar el impacto real de la violación de normalidad.

El mé todo fue fácilmente codificable en MS Excel, por lo que permitirí a distribuirlo de una manera conveniente a las personas pertinentes de la compañía, sin necesidad de adquirir paqueterí a computacional adicional. El mismo problema de planeación resuelto por programación matemática entera, habría excedido la capacidad del optimizador básico incluido en MS Excel.

\section{Escenarios de prueba para el modelo de nivel de servicio}

Los resultados iniciales de este trabajo ayudaron a determinar la factibilidad de las ideas presentadas a través de un caso real; sin embargo, es siempre de valía probar varios escenarios posibles para evaluar el desempeño del mé todo. Por cuestiones de espacio, aquí presentamos solamente dos escenarios que resultaron interesantes: en el primero se alimentan datos con un pico de demanda, precedido de una caída de la misma; y en el segundo, se presenta una instancia con dos picos de demanda en períodos no consecutivos en el mismo horizonte de análisis.

En el primer caso, la capacidad inicial de la red es de 348 unidades y la demanda en el primer mes es de 300 unidades. La demanda va creciendo moderadamente hasta el quinto mes, donde ésta cae a 200 unidades seguida por un crecimiento abrupto en el siguiente período que la lleva a un nivel de 510 unidades de ancho de banda. Los resultados con el modelo de nivel de servicio a un nivel de seguridad de $85 \%$ pueden verse en la figura 6. Es importante notar que, aunque el inventario se reduce significativamente, el nivel de servicio aún pudo proveer protecció $n$ contra el no cumplimiento de la demanda.

El segundo caso se presenta en la figura 7, donde dos picos de demanda suceden en períodos no consecutivos dentro de un mismo horizonte de planeación de 12 períodos. La protección, como se puede ver, nuevamente resulta eficaz.

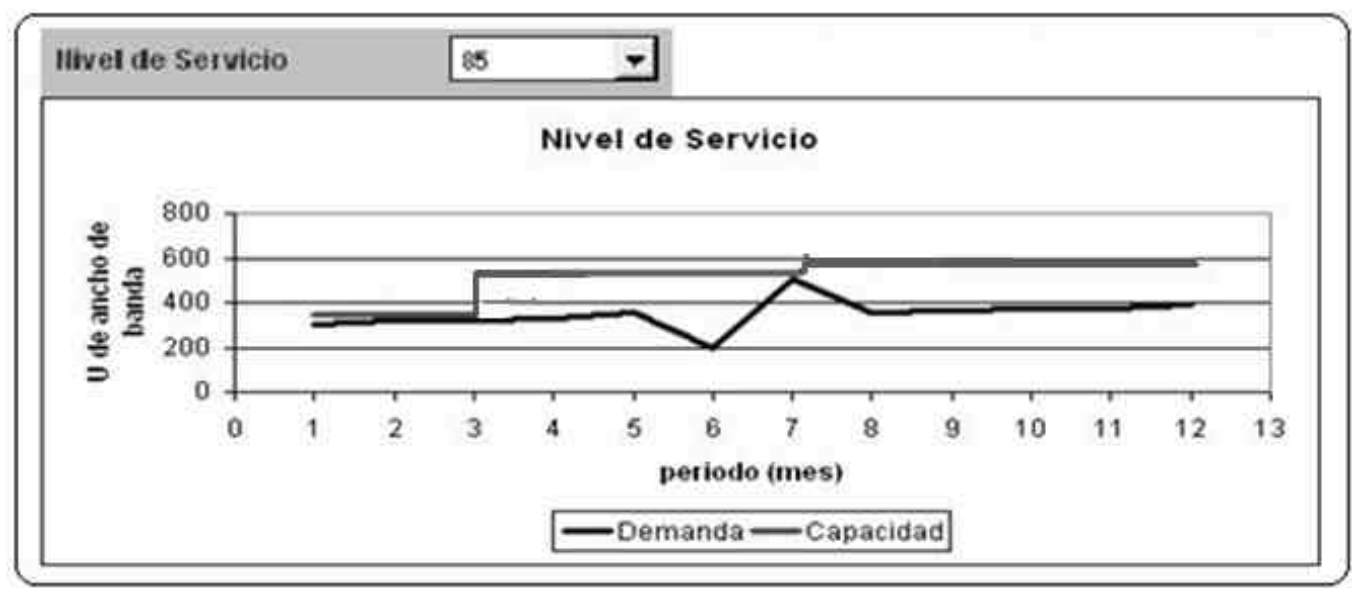

Figura 6. Gráfica de resultados para el crecimiento súbito de la demanda de un mes a otro 


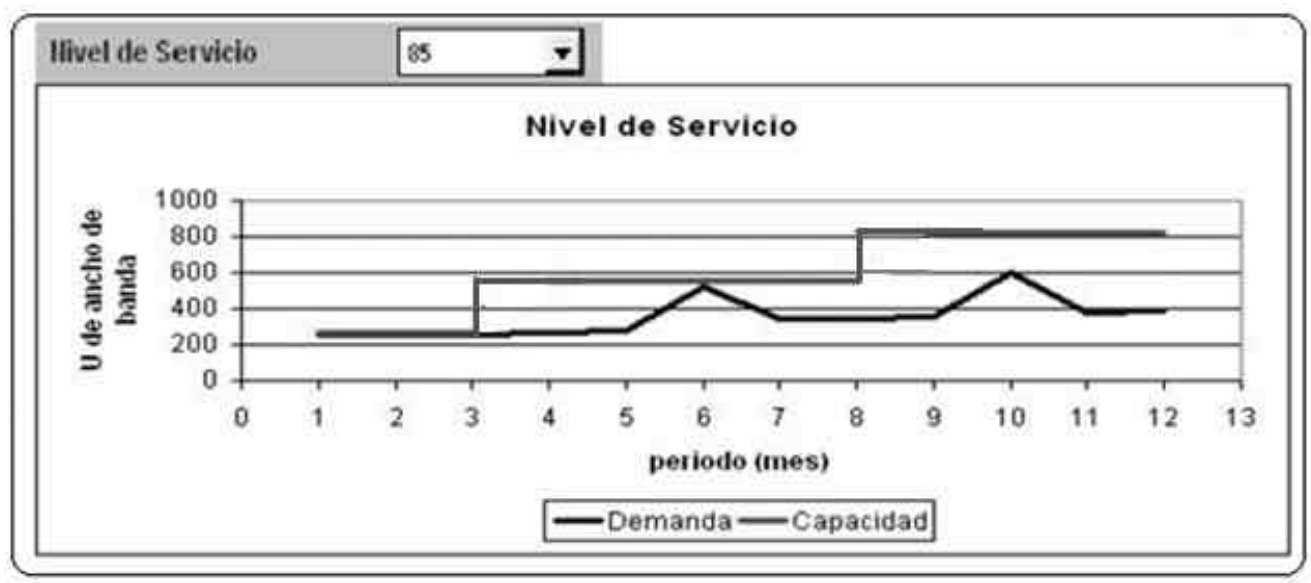

Figura 7. Gráfica de resultados para el crecimiento extremo de la demanda de un mes a otro

Dada la dependencia de la planeación de capacidad en la calidad de los pronó sticos disponibles de demanda, la habilidad del método de ajustar el nivel de servicio, así como su capacidad de proveer protección ante fluctuaciones como é stas, resultan características muy atractivas para su aplicación en la industria.

\section{Conclusiones}

En este trabajo se demostró cómo utilizar un enfoque de control de inventarios para un problema de planeación de capacidad en redes de telecomunicaciones. Tal enfoque resultóen soluciones que se pueden generar de manera muy eficiente.

Las técnicas de control de inventarios fueron fácilmente implementadas en hojas de cálculo de MS Excel, lo que implicó que no se necesitaría invertir en paquetería computacional especializada, facilitando así la distribución y el uso de los modelos en una compañía.

Se mostró también que el método propuesto puede ser de gran utilidad para soportar fallas potenciales de pronóstico de demanda cuando se requiere proveer un nivel alto de servicio.
Finalmente, los resultados subrayan la importancia de establecer similitudes entre problemas de distintas áreas para capitalizar en las técnicas de solución correspondientes, y por supuesto, apuntan a favor del trabajo interdisciplinario.

\section{Agradecimientos}

Al personal de la empresa de telecomunicaciones, quienes prefieren permanecer en el anonimato, por el apoyo brindado durante este proyecto.

\section{Referencias}

Alvarez-Herrera C.A. y Cabrera-Ríos M. (2005). Un enfoque de inventarios para planear capacidad en redes de telecomunicaciones. Ingenierías, 8:29, pp. 59-65.

Coyle A. (1998). An Algorithm for Capacity Expansion in Local Access Networks. Proceedings of the $33^{\text {rd }}$ Annual Conference of the Operations Research Society of New Zealand.

Solow D. y Mathur K. (1996). Investigación de Operaciones. Editorial Prentice-Hall, Mexico, pp. 637-699.

Sprangins J.D., Hammond J.L. and Pawlikowski K. (1992). Telecommunications, Protocols and Design. Editorial 
DOI: http://dx.doi.org/10.22201/fi.25940732e.2007.08n4.019

Control de inventarios y su aplicación en una compañía de telecomunicaciones

\author{
Addison-Wesley, California, EE.UU., \\ pp. $105-113$
}

\title{
Semblanza de los autores
}

Carlos Alberto Alvarez-Herrera. Es ingeniero administrador de sistemas y maestro en ciencias en ingeniería de sistemas por la Universidad Autónoma de Nuevo León (UANL). Actualmente trabaja en el área de control de inventarios para una compañía de electrodomésticos y línea blanca.

Mauricio Cabrera-Ríos. Es ingeniero industrial y de sistemas por el ITESM, Campus Monterrey. Asimismo, es maestro en ciencias y doctor en ingeniería indus trial y de sistemas por The Ohio State Univer sity en Columbus, Ohio. Actualmente es profesor investigador del Posgrado en Ingeniería de Sistemas de la FIME, UANL. Sus líneas de investigación abarcan la caracterización, la modelación y la optimización de procesos de manufactura y servicios. 